Sección uno: Ensayo

Radiografía de la innovación educativa en el Siglo XXI

\title{
El Aprendizaje y Servicio (ApS) en la enseñanza del alemán: una propuesta innovadora para la Escuela Oficial de Idiomas ${ }^{1}$
}

Service Learning (S-L) in German teaching: an innovative proposal for the Official State Language School

Lucía-Pilar Cancelas-Ouviña

Universidad de Cádiz

lucia.cancelas@uca.es

Ana Hurtado-Sierra

El Centro Inglés

anahursie@gmail.com

\section{Resumen}

En este artículo mostramos como puede adaptarse la metodología innovadora de aprendizaje y servicio (ApS) a la enseñanza de Idiomas. Presentamos un proyecto de innovación educativa diseñado para ser implementado en una relación de partenariado entre el alumnado de $5^{\circ}$ curso de la Escuela Oficial de Idiomas de Chiclana (Cádiz) que cursa alemán nivel B2.2 y la Asociación Fórum Germano-gaditano con sede en El Puerto de Santa María (Cádiz) con el objetivo de acercar la cultura española a hablantes germanos a través del Museo o Centro de Interpretación del Vino y la Sal de Chiclana de la Frontera (Cádiz). Este ApS daría respuesta a la necesidad real de integración de un colectivo de inmigrantes germano hablantes que quieren socializarse y conocer la cultura de su país de acogida.

Palabras Clave: Innovación Docente, Aprendizaje y Servicio, Escuela Oficial de Idiomas, Aprendizaje de Idiomas, Migración

${ }^{1}$ Recibido: 02/02/2020 Evaluado: 19/02/2020 Aceptado: 26/02/2020 


\begin{abstract}
In this article we show how the innovative methodology of Service Learning (S-L) can be adapted to language teaching. We present an innovative proposal designed to be implemented through a partnership between the students of the 5th year of the Chiclana Official State Language School who study German level B2.2 and the German Association Fórum Germano-gaditano based in El Puerto de Santa María (Cádiz), in order to bring German speakers closer to Spanish culture at the Centro de Interpretación del Vino y la Sal in Chiclana de la Frontera (Cádiz). This Service Learning responds to a real integrational need of a group of German-speaking immigrants who want to socialize and learn about their host country's culture.
\end{abstract}

Key Words: Educational Innovation, Service Learning, Official State Language School, language learning, migration

\title{
Introducción
}

En la actualidad existen múltiples metodologías innovadoras que se ponen en práctica en las aulas con el fin de mejorar el proceso de enseñanza-aprendizaje del alumnado. De todas las tendencias en innovación educativa que están en boga hoy en día hemos elegido el Aprendizaje y Servicio (ApS) como metodología que sustentará la propuesta de innovación educativa aplicada al aprendizaje de idiomas que presentamos en este artículo

Tras consultar las bases de datos de proyectos, experiencias y buenas prácticas de ApS más relevantes de España (Red española de Aprendizaje Servicio (https://aprendizajeservicio.net/), Fundación Zerbikas (http://www.zerbikas.es/), Centro Promotor de Aprendizaje Servicio (https://aprenentatgeservei.cat/), nos encontramos en los últimos años con una gran cantidad de proyectos $\mathrm{ApS}$ de diversa temática provenientes del ámbito universitario. Así mismo, son cada vez más frecuentes las propuestas ApS vinculadas con centros de educación primaria y secundaria. Sin embargo, dado que los proyectos ApS vinculados con las Escuelas Oficiales de Idiomas (en adelante EOI) son escasos o apenas inexistentes vimos la necesidad de mostrar una propuesta centrada en este tipo de enseñanza de régimen especial que se imparte en toda España.

En este artículo ejemplificaremos como puede implementarse esta metodología presentando un servicio real y auténtico diseñado para ser desarrollado en un marco de reciprocidad entre el alumnado de $5^{\circ}$ curso de la Escuela Oficial de Idiomas de Chiclana (Alemán B2.2) y la Asociación Fórum Germano-gaditano.

Comenzaremos abordando los referentes teóricos del ApS. Proseguiremos explicando el contexto para el que hemos diseñado este proyecto innovador poniendo el foco en los miembros de la EOI que ofrecen el servicio y en el Fórum germano-gaditano como 
potenciales beneficiarios. Posteriormente, detallamos el servicio que va a ofrecerse reflejando aspectos tales como los objetivos de aprendizaje, las fases del proyecto, la temporalización y la evaluación entre otros.

Por último, hacemos una reflexión sobre la idoneidad y el gran potencial de la aplicación de esta metodología innovadora en las EOIs ofreciendo una experiencia que puede ser fácilmente replicable adaptándola a distintas asociaciones y colectivos de inmigrantes o residentes extranjeros que necesiten integrarse en la sociedad española y cuyo proceso de adaptación se verá ampliamente favorecido por el conocimiento de la cultura y de la lengua del país de acogida. A su vez el alumnado que ofrece el servicio está desarrollando la competencia comunicativa utilizando la Lengua Extranjera objeto de estudio en situaciones reales con hablantes nativos.

\section{Qué es el Aprendizaje y Servicio (ApS)}

El Aprendizaje y Servicio (en inglés Service Learning S-L) es una metodología educativa que combina los procesos de aprendizaje del alumnado con la prestación de servicios a la comunidad. Se la conoce con las siglas ApS (aprendizaje-servicio) o AySS (aprendizaje y servicio solidario). En palabras de Batlle, (s.f.) el ApS es una propuesta educativa que vincula el aprendizaje con un servicio a la comunidad con el objetivo de paliar necesidades reales del entorno. Batlle (s.f.) añade: "lo que hace el aprendizaje-servicio es completar la acción solidaria con el vínculo curricular, ofreciendo a los alumnos la oportunidad de aprender siendo útiles a los demás" (p.4).

El ApS es un enfoque con un marcado carácter experiencial sustentado en la metodología "Learning by doing" (aprender haciendo) (Domingo y Balaguer, 2017) donde la realización de actividades y tareas auténticas y reales juegan un rol esencial. Diseñamos un programa basado en un enfoque de trabajo por tareas donde el aprendiz adquiere y construye una serie de contenidos gracias al desarrollo de un proyecto.

El valor añadido del ApS (Gezuraga \& García-Pérez, 2018) radica en ser un Aprendizaje por Proyectos con fin solidario, y desde ahí es donde confluyen dos intencionalidades tradicionalmente entendidas desde la dicotomía de lo académico y la social. En lo que coinciden la mayoría de los autores, es que se trata de un proyecto bien articulado (Puig et al., 2007). Por tanto, es esencial considerar la organización del proyecto, que debe estar planificado y relacionado con el currículo (Mayor, 2013).

La autora Xus Martín (2015), destaca que "el núcleo del aprendizaje servicio está formado por la articulación de tres dinamismos educativos básicos: las necesidades o retos cívicos, el servicio a la comunidad y el aprendizaje de contenidos, competencias y valores” (p.29).

Martínez Lozano y otros (2018) añaden que el ApS "propicia el desarrollo de competencias sociales y cívicas, y el ejercicio activo de la ciudadanía, entre aquellos que la practican" (p.11). 
Como podemos observar, el término "servicio" es clave en esta metodología, entendiéndose este como un conjunto de tareas que se realizan altruistamente para mitigar una necesidad (Puig et al., 2015).

Para terminar de comprender esta metodología innovadora, contemplaremos ciertos elementos básicos de los procesos basados en ApS (García Ortiz, 2013):

1. Debe estar basado en un proyecto bien planificado.

2. No se trata de un proceso de beneficencia ni de paternalismo.

3. Existe un claro protagonismo de los estudiantes.

4. Requiere una reflexión continua durante todo el proceso y al final del mismo.

García Ortiz (2013) aporta un esquema (Imagen 1) que sintetiza los elementos que componen el ApS, coincidiendo casi en la totalidad con los autores anteriormente mencionados

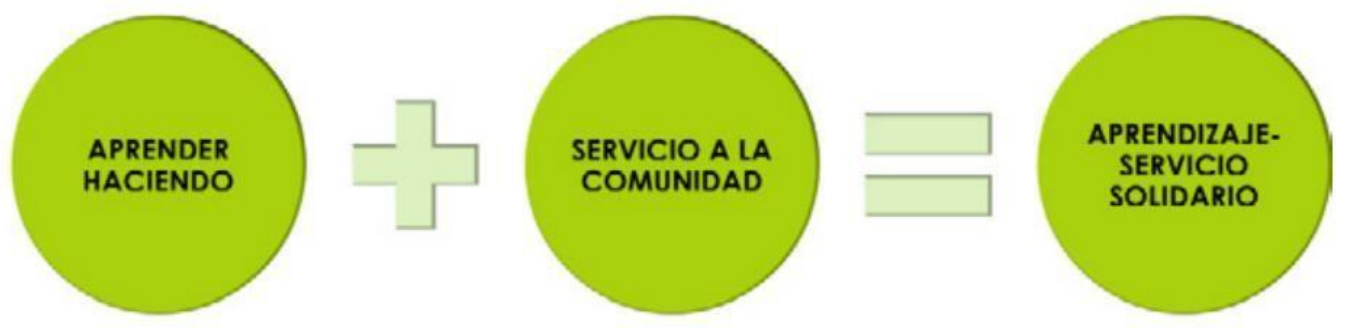

Imagen 1: Qué es el aprendizaje servicio. Fuente: García Ortiz (2013)

Chupp y Joseph (2010) identifican dentro de la metodología ApS cuatro tipos: el ApS tradicional, el ApS como justicia social, el ApS crítico y el ApS de transformación institucional. García-Romero y Lalueza (2019) a su vez clasifican los trabajos ApS en cuatro grandes modelos teóricos atendiendo a los procesos de aprendizaje que se dan: Aprendizaje Experiencial, Aprendizaje Transformativo, Pedagogía Crítica y Teoría de la Actividad.

En este trabajo nos decantaremos por un ApS tradicional de aprendizaje experiencial donde el foco son los estudiantes y se persigue su aprendizaje, mientras que el ámbito de servicio es el de "Ayuda directa a otras personas". El servicio comunitario mejora el aprendizaje académico a través de la acción, la reflexión y la aplicación. La experiencia y la exposición al mundo real mejora la retención y la aplicación de los contenidos del curso (Gezuraga y García-Pérez, 2018). Dentro de las competencias cívicas y sociales que pretendemos desarrollar con este proyecto están: luchar contra los estereotipos, fomentar el respeto hacia otras lenguas y culturas, favorecer la integración de migrantes y promover la aceptación de personas procedentes de otros países. 


\section{Relación de partenariado: EOI y Asociación Fórum Germano-gaditano}

El proyecto ApS que queremos promover establecerá una relación de partenariado entre el alumnado de la EOI de Chiclana de la Frontera y los miembros de la Asociación Fórum Germano-gaditano de la Provincia de Cádiz beneficiando igualmente a los destinatarios y a los estudiantes que prestarán el servicio. Las dos partes implicadas reciben algo valioso: los estudiantes aprenden al prestar el servicio y realizar y los miembros de la Asociación verán cubierta una necesidad que ellos manifiestan.

Entendemos por partenariado la "colaboración entre dos o más instituciones sociales y educativas independientes orientada a la realización conjunta de una actividad. El partenariado en el aprendizaje servicio vincula las entidades con el propósito de desarrollar una actividad educativa dando respuesta a necesidades sociales." (Puig 2015, p.6).

Este ApS permitirá al alumnado de la EOI entrar en contacto con una comunidad cultural, social y lingüísticamente distinta a la que ellos pertenecen utilizando el alemán como lengua vehicular para dar a conocer la cultura española con el objeto de facilitar la integración en nuestro país del colectivo de residentes de habla alemana.

Pasamos a analizar a los participantes de esta experiencia ApS para tener una visión más clara de las necesidades y de los objetivos de aprendizaje.

\section{Asociación Fórum Germano-gaditano: detección de necesidades.}

En la actualidad hay una comunidad de 167.901 alemanes (González Enríquez, 2019) residentes en España que están asentados en su mayoría en localidades de la Comunidad Valenciana, Costa del Sol, Costa de la Luz, Islas Canarias y Baleares. Suelen buscar el buen clima, el sol y la playa y muchos de ellos tienen como destino España tras su jubilación, aunque otros están integrados en España desempeñando todo tipo de profesiones. En las ciudades o localidades de acogida suelen crear colonias, grupos o asociaciones viviendo, en muchas ocasiones, en una burbuja donde solo se relacionan con sus compatriotas con los que comparten lengua, cultura, ocio e intereses y que les lleva en ocasiones a situaciones de cierto aislamiento.

Realizamos un mapeo del entorno para rastrear qué asociaciones, entidades sociales, instituciones o colectivos de inmigrantes tienen necesidades de integración y de establecer vínculos con miembros de la sociedad española. En este rastreo encontramos que en la provincia de Cádiz existe una población importante de alemanes que han constituido la Asociación Fórum Germano-gaditano de la provincia de Cádiz. Sus miembros son germano hablantes que residen o residirán dentro en breve en la provincia y que se reúnen periódicamente para mantener el contacto entre ellos, recibir ayuda, realizar actividades de ocio, integrarse en nuestro país, socializarse, etc.

La asociación cuenta con una página web (https://forum-germano-gaditano.com) que se actualiza con regularidad donde se publican noticias relacionadas con los intereses del Fórum y entradas con los lugares y fechas de los encuentros que mantienen mensualmente, así como otras actividades programadas por la Asociación. 


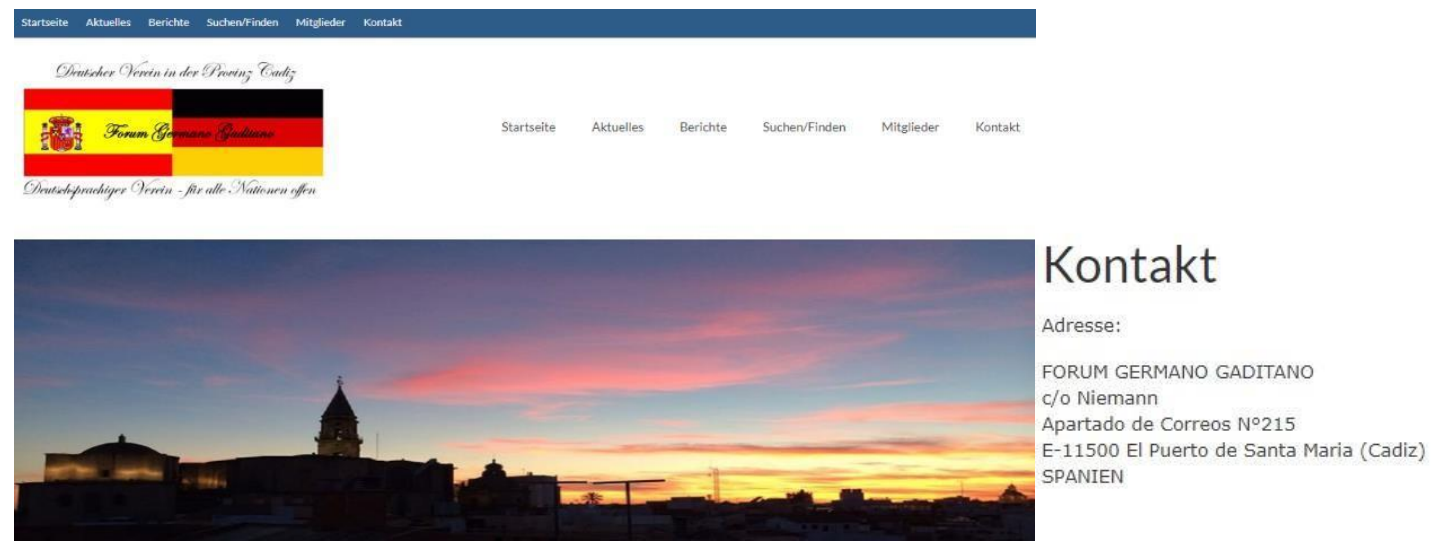

Imagen 2: Página web de la asociación Fórum Germano-gaditano.

Como observamos en su página web (Imagen 2), pretenden participar en la vida cultural y política del país de acogida a la vez que intentan acercar la cultura de sus países de origen a los españoles y establecer vínculos entre ellos que fomenten la integración.

Entre sus objetivos destacan fomentar la relación germano-española participando en eventos españoles e invitando a españoles a participar en eventos de la asociación, además de establecer áreas de interés conjuntas y desarrollarlas en temas como son la cultura, historia, lengua y temas legales.

Teniendo en cuenta esta presentación en su página web detectamos una necesidad clara de integración y de creación de vínculos con la sociedad española planteada por ellos, y llegamos a la conclusión de que es una entidad idónea con la que establecer un proyecto ApS para estudiantes de alemán como lengua extranjera porque, podrán ayudarse mutuamente a aprender y enriquecerse sobre temas de interés comunes entre ambos grupos.

\section{La EOI ofreciendo un servicio a la comunidad}

La Escuela oficial de idiomas es una institución fundada en 1911 (Monterrey 2003), que ofrece una enseñanza permanente de régimen especial centrada exclusivamente en la enseñanza de lenguas modernas. España es el único país de la Unión Europea que dispone de una institución pública de nivel no universitario dedicada a la enseñanza especializada de idiomas dependiente de las Consejerías de Educación de las diferentes Comunidades Autónomas. Dispone de una red extensa de 305 EOIs que ofrecen formación en 23 idiomas y que expide certificados de estudios cualificadores acordes a los niveles del Marco Común de Referencia Europeo de las Lenguas (MCREL). (Cancelas y Ouviña, 2014).

Uno de los principales problemas con el que se enfrentan las EOIs a nivel internacional es la falta de reconocimiento de los certificados que expiden, a diferencia de otras instituciones 
como la Universidad de Cambridge, la Alianza Francesa, el Instituto Goethe, el Instituto Cervantes, etc. (Araujo, 2018).

El alumnado y la docencia de la lengua extranjera en las EOIs reúnen unas características particulares, que han sido analizadas por diversos autores (Díaz Ortega, 2005; Corredera, 2005 y Cancelas y Ouviña, 2014). El alumnado suele ser bastante heterogéneo en cuanto a edad, formación y profesión (Díaz Ortega, 2005). Normalmente, se trata de alumnado adulto y en el caso de los adolescentes (mayores siempre de 14 años) tienden a comportarse con gran madurez condicionados por el entorno en el que se encuentran. Es necesario que tengamos en cuenta que el aprendizaje adulto entraña ciertas peculiaridades en cuanto a motivación, capacidades, modalidades de conducta, actitud hacia el tiempo de los sujetos y todo ello condiciona el tipo de relación docente-estudiante que se da en el aula Escobar, 1978).

Además, el alumnado de las EOIs suele acudir a clase con expectativas y objetivos claros, algunos arrastran malas experiencias personales debido a las metodologías con las que aprendieron idiomas en el pasado y normalmente tienen más dificultades para expresarse a nivel oral debido al miedo al ridículo, a equivocarse o a no conseguir los objetivos que se habían propuesto (Cancelas y Ouviña, 2014).

Corredera (2005) incide en la necesidad de contar con docentes creativos en las EOIs, capaces de atender a las diversas necesidades propias de estos centros. Sin embargo, destaca la ausencia de literatura científica relacionada con la innovación docente que se lleva a cabo en las escuelas de idiomas y que son indispensables para acercarse al modelo de docente innovador que propone Corredera (2005).

Este alumnado adulto puede tener unas ideas preconcebidas sobre la cultura de los hablantes de la lengua objeto de estudio que se han ido forjando a lo largo de toda su vida y que en muchas ocasiones no tiene por qué corresponder con la realidad. Este ApS les permitirá entrar en contacto con hablantes reales que les mostrarán el contexto cultural de su país desterrando prejuicios e ideas erróneas.

Con este trabajo queremos dar respuesta a la poca presencia de propuestas de innovación educativa para las EOIs proponiendo un proyecto de ApS que vendría, en parte, a cubrir carencias en la cultura innovadora de estos centros. Por añadidura, el aprendiz adulto encontrará sentido a lo que estudia cuando es capaz de aplicar sus conocimientos y habilidades en una situación real e irá adquiriendo seguridad en sí mismo cuando se expresa en una lengua extranjera.

\section{Propuesta didáctica innovadora: ApS en la EOI de Chiclana: acercamiento de la cultura andaluza a la asociación Fórum Germano-gaditano}

\section{Identificación de los sujetos que ofrecen el ApS}

El servicio será ofrecido por 25 estudiantes de edades y formación diversa dentro del marco de la materia Alemán Lengua Extranjera, Nivel B2.2 de la Escuela Oficial de Idiomas. 


\section{Temporalización y calendario}

Este proyecto de ApS se llevará a cabo durante el último trimestre del curso académico para activar y aplicar todos los conocimientos adquiridos durante todo el año. Constará de cuatro sesiones de 135 minutos cada una donde se prestará el servicio. Además, el alumnado dispondrá de 3 sesiones adicionales de 135 minutos que se integrarán en las distintas unidades didácticas que se estén impartiendo en la EOI durante este tercer trimestre para preparar el servicio que se va a prestar trabajando y produciendo los textos escritos en alemán y para preparar las exposiciones orales.

Para adaptarnos y seguir la dinámica de encuentros y actividades que promueve la entidad social en su página web, les prestaremos el servicio con una periodicidad mensual entre marzo y junio consensuando previamente las fechas de encuentro.

\section{Objetivos específicos marcados para este proyecto}

Nos hemos planteado para este proyecto de ApS los siguientes objetivos de aprendizaje clasificándolos en dos categorías:

- Objetivos específicos del Servicio:

1. Facilitar la integración de ciudadanos germano hablantes en nuestro país.

2. Dar a conocer la cultura española a ciudadanos germano hablantes utilizando el alemán como lengua vehicular.

3. Dar a conocer la cultura andaluza a través del Centro de Interpretación del Vino y la Sal de Chiclana de la Frontera.

4. Acabar con la barrera idiomática que encuentran los visitantes extranjeros cuando acuden a museos y centros culturales y no disponen de información en su lengua materna para poder entender y apreciar los productos culturales que están viendo.

5. Actuar como mediadores e intérpretes creando material visual y audiovisual en alemán.

6. Crear un blog en alemán para difundir las tareas y actividades vinculadas con el proyecto de ApS.

7. Crear tándems lingüísticos para promover la competencia comunicativa del alumnado.

8. Intercambiar correos electrónicos formales e informales.

9. Crear un glosario español>alemán con el vocabulario específico de la unidad.

10. Crear un guion-resumen de las exposiciones.

11. Diseñar y elaborar material de apoyo destinado a germano hablantes.

12. Hacer presentaciones formales claras y detalladas sobre los temas que presentarán en el Centro de Interpretación del Vino y la Sal.

13. Promover lazos de unión y cooperación entre los miembros de la Asociación Fórum Germano-gaditano y el alumnado de alemán de la EOI a través de la creación de tándems. 
14. Fomentar la relación germano-española promoviendo el respeto, la tolerancia y la erradicación de la xenofobia y estereotipos asociados a miembros de distintas culturas.

15. Establecer áreas de interés conjuntas y desarrollar sinergias entre ambas instituciones en temas vinculados con la lengua, la cultura, la historia y la literatura española y germana.

- Objetivos de aprendizaje del alumnado que presta dicho servicio:

1. Contribuir a la consolidación de la competencia lingüística en alemán en el alumnado de la EOI.

2. Utilizar la lengua extranjera en situaciones reales fuera del aula.

3. Escribir y comunicarse con corrección en el blog creado para el proyecto.

4. Mejorar la seguridad y autoestima del alumnado gracias al ofrecimiento de un servicio real a hablantes nativos.

5. Mejorar la competencia digital del alumnado creando recursos digitales y utilizando redes sociales para comunicarse en la lengua extranjera.

6. Comprender ideas principales de una conversación informal con miembros del Fórum Germano-gaditano sobre la situación turística y gastronómica en Alemania y en la provincia de Cádiz.

7. Participar activamente en conversaciones y discusiones informales con uno o más miembros del Fórum Germano-gaditano.

8. Comprender ideas principales y detalles más relevantes de correos electrónicos escritos por miembros del Fórum sobre curiosidades culturales de Alemania.

9. Tomar notas en alemán sobre el contenido de las exposiciones de los compañeros realizadas en el Centro de Interpretación del Vino y la Sal.

10. Crear un portafolio con detalles del proceso del proyecto, las actividades propuestas y los conocimientos adquiridos.

\section{Fases de la propuesta innovadora ApS}

La propuesta ApS que presentamos tiene un carácter sistémico y está inspirada en Alcaraz, Chaparro y García (2016) y Mayor Paredes (2013) y está conformada por las siguientes fases:

\begin{tabular}{|c|c|c|}
\hline Fases del ApS & Descripción de las actuaciones & Agentes \\
\hline FASE 1. & Identificar: & \\
\hline $\begin{array}{l}\text { Identificación de } \\
\text { necesidades }\end{array}$ & $\begin{array}{l}\text { 1.1. Necesidades curriculares del alumnado de la } \\
\text { EOI. } \\
\text { Se tendrá en cuenta: } \\
\text { - Currículo oficial. } \\
\text { - Programación didáctica de la EOI. } \\
\text { - Características particulares del grupo clase } \\
\text { que participará en el proyecto. }\end{array}$ & $\begin{array}{l}\text { Docentes } \\
\text { que } \\
\text { promueven } \\
\text { el ApS }\end{array}$ \\
\hline
\end{tabular}




\begin{tabular}{|c|c|c|}
\hline Fases del ApS & Descripción de las actuaciones & Agentes \\
\hline & $\begin{array}{l}\text { 1.2.Necesidades de los miembros de la Asociación } \\
\text { Fórum Germano-gaditano. } \\
\text { - Identificadas a partir de lo descrito en su } \\
\text { página web donde recogen la finalidad y } \\
\text { objetivos de la Asociación. }\end{array}$ & \\
\hline $\begin{array}{l}\text { FASE } 2 . \\
\text { Establecer la } \\
\text { cooperación entre } \\
\text { EOI y la Asociación } \\
\text { Fórum Germano- } \\
\text { gaditano }\end{array}$ & $\begin{array}{l}\text { 2.1. Establecimiento de alianzas entre ambas } \\
\text { instituciones. } \\
\text { La propuesta debe promover un entorno de } \\
\text { aprendizaje colaborativo y cooperativo entre el } \\
\text { Equipo Directivo y docente de EOI y la Junta } \\
\text { Directiva de la Asociación. }\end{array}$ & $\begin{array}{l}\text { EOI y } \\
\text { Asociación }\end{array}$ \\
\hline $\begin{array}{l}\text { FASE } 3 . \\
\text { Concreción del } \\
\text { servicio a la } \\
\text { Asociación }\end{array}$ & $\begin{array}{l}\text { 3.1. Ambas instituciones deberán acordar las } \\
\text { actuaciones que se llevarán a cabo y programar y } \\
\text { consensuar las fechas de las actividades. }\end{array}$ & $\begin{array}{l}\text { Equipo } \\
\text { Docente y } \\
\text { Junta } \\
\text { Directiva } \\
\text { de la } \\
\text { Asociación }\end{array}$ \\
\hline
\end{tabular}

\section{FASE 4.}

Concreción de aprendizajes académicos
4.1. Estableceremos los objetivos de aprendizaje y sus vinculaciones curriculares concretando competencias, contenidos, actividades, recursos, Docentes etc. que contemplaremos en este proyecto.

\section{FASE 5.}

Plan de acción y ejecución del proyecto

(Subdivido en subfases)

\subsection{Presentación del Proyecto:}

- Presentación del Proyecto a los participantes.

- Encuentro entre el alumnado y los miembros de la Alumnado Asociación.

EOI

- Presentación del blog que nos servirá como eje Miembros vertebrador de nuestra propuesta. asociación

- Conformación de 8 equipos de trabajo con el alumnado de la EOI y asignación de tareas y roles.

- Designación de tándems lingüísticos. Cada alumno/a tendrá asignada una pareja del Fórum.

El tándem lingüístico es un sistema de aprendizaje autónomo de la Lengua Extranjera en pareja donde cada estudiante se empareja con un nativo de la lengua meta objeto de estudio y ambos realizan 


\begin{tabular}{|c|c|c|}
\hline Fases del ApS & Descripción de las actuaciones & Agentes \\
\hline & $\begin{array}{l}\text { tareas tanto presenciales como virtuales para } \\
\text { mejorar su competencia lingüística y cultural. Es } \\
\text { una relación simbiótica y cooperativa donde se } \\
\text { benefician ambos miembros del tándem que } \\
\text { autogestionan su aprendizaje alternando las } \\
\text { lenguas de ambos en las interacciones. }\end{array}$ & \\
\hline & 5.2. Fase de documentación: & \\
\hline & $\begin{array}{l}\text { Cada equipo de trabajo de la EOI se documentará } \\
\text { buscando información sobre el tema asignado que } \\
\text { deberán presentar en alemán a los miembros de la } \\
\text { Asociación. }\end{array}$ & $\begin{array}{l}\text { Alumnado } \\
\text { EOI }\end{array}$ \\
\hline & 5.3. Fase de elaboración de materiales & \\
\hline & $\begin{array}{l}\text { Diseño y creación de cartelería y materiales de } \\
\text { apoyo para las exposiciones en alemán. }\end{array}$ & \\
\hline & $\begin{array}{l}\text { 5.4. Exposiciones en el Centro de interpretación } \\
\text { del vino y la sal }\end{array}$ & $\begin{array}{l}\text { Alumnado } \\
\text { EOI }\end{array}$ \\
\hline & $\begin{array}{l}\text { Los distintos grupos de alumnado de la EOI } \\
\text { actuarán de Guías Turísticos con los miembros de } \\
\text { la Asociación Fórum Germano-Gaditano en el } \\
\text { Centro de interpretación del vino y la sal. }\end{array}$ & $\begin{array}{l}\text { Alumnado } \\
\text { EOI }\end{array}$ \\
\hline & $\begin{array}{l}\text { 5.5. Durante la ejecución del proyecto } \\
\text { registraremos lo que vamos haciendo (fotografías, } \\
\text { vídeos, etc...) y dentro de lo posible haremos una } \\
\text { campaña de comunicación (invitar a la prensa, } \\
\text { publicar en la web del centro...) para reforzar el } \\
\text { compromiso del grupo. }\end{array}$ & asociación \\
\hline
\end{tabular}

Alumnado

EOI

Alumnado

EOI

Alumnado

Miembros

asociación

FASE 6.
Reflexión de la
acción y cierre del
proyecto con el
grupo.

\section{FASE 6.}

Reflexión de la proyecto con el grupo.
Esta fase es especialmente importante. Docentes Mantendremos encuentros dialógicos donde todos los participantes en el ApS valorarán el desarrollo de la experiencia y analizarán los aprendizajes conseguidos promoviendo la reflexión y valorarán Miembros el impacto del servicio. 


\begin{tabular}{llll}
\hline Fases del ApS & Descripción de las actuaciones & Agentes \\
\hline FASE 7. & $\begin{array}{l}\text { El proyecto y los resultados obtenidos se } \\
\text { divulgarán por todos los canales de comunicación } \\
\text { Divulgación del } \\
\text { Proyecto ApS }\end{array}$ & Docentes \\
& - & \\
& Todas las tareas realizadas se divulgarán a & \\
& través del Blog elaborado para el proyecto: & Alumnado \\
& https://ldewillkommeninspanien.blogspot.com/ & EOI \\
& Página web de la Asociación Fórum & \\
& Germano-gaditano. & Miembros \\
& La página web de la EOI. & Asociación.
\end{tabular}

FASE 8.

Evaluación de la propuesta
Al finalizar el ApS se realizará un debate con todos los implicados sobre el desarrollo y utilidad del Proyecto realizando una evaluación multifocal donde analizaremos tres niveles:

1.Evaluaremos al grupo y a sus miembros,

2.Evaluaremos el trabajo en red con las entidades.

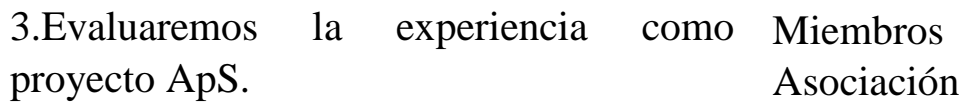

Además, el proceso de evaluación se concretará con los instrumentos y estrategias diferenciadas para cada uno de los agentes implicados en el proyecto que especificamos a continuación:

\section{*Alumnado EOI:}

- Debate de reflexión final sobre la experiencia.

- Informe de Autoevaluación.

- Cuestionario sobre el proyecto ApS.

- Prueba objetiva en alemán sobre los contenidos para comprobar que se han logrado los objetivos didácticos $\mathrm{y}$ competencias vinculadas con la asignatura Alemán Lengua Extranjera.

- Evaluación de las producciones orales y escritas del alumnado.

- Portafolio elaborado individualmente vinculado con el proyecto.
Alumnado
EOI

Docentes Asociación 


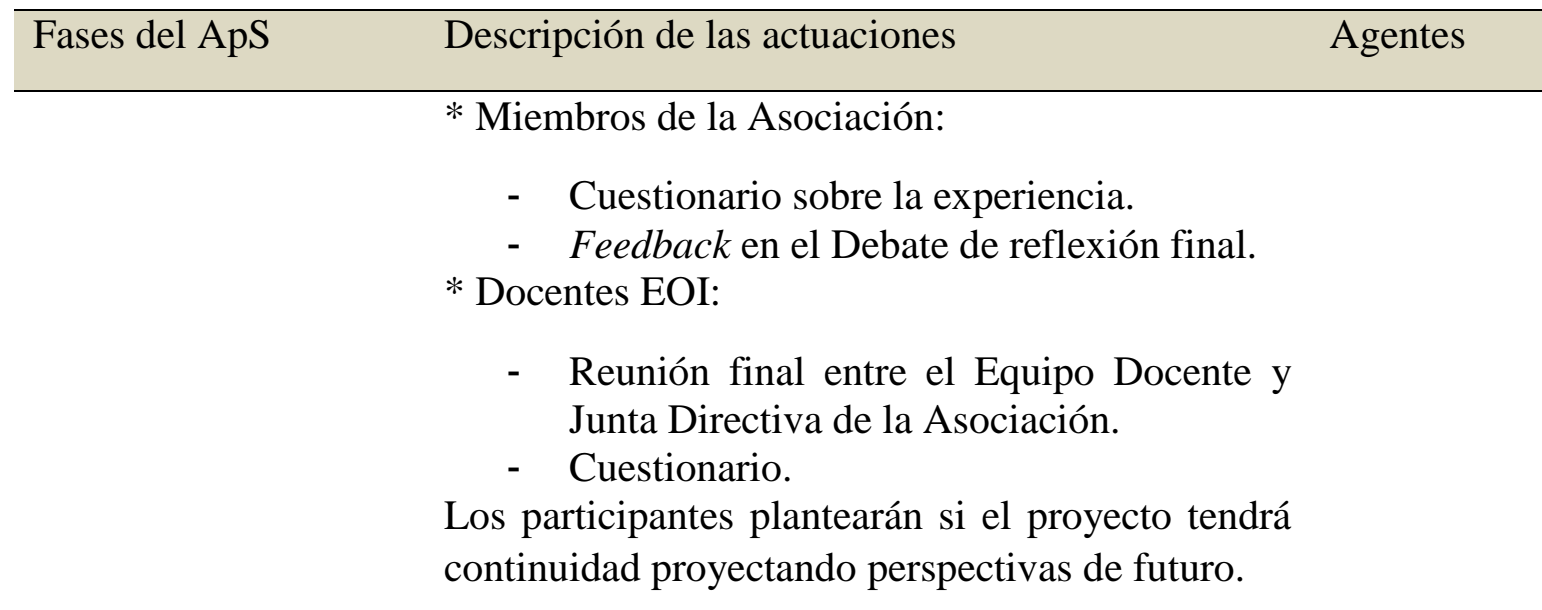

FASE 9. Conclusiones
Las conclusiones se recogerán en una Memoria Final que elaborará el Equipo Docente de la EOI donde incluirán todas las evidencias de la experiencia, los aprendizajes conseguidos, las Equipo Docente dificultades, los puntos débiles y fuertes del proyecto y los datos obtenidos a partir de la evaluación realizada en la fase 8 .

Tabla 1: Fases del ApS. Elaboración propia a partir de Mayor Paredes (2013) y Alcaraz, Chaparro y García (2016).

5. ¿Qué servicios se ofrecen en este ApS?

La Asociación Fórum Germano-gaditano demanda salidas y visitas culturales para integrarse y entrar en contacto con la cultura española con una periodicidad de una actividad mensual. En los museos y centros culturales los rótulos, paneles y material audiovisual suelen ofrecer información prioritariamente en la lengua vernácula del país por lo que los visitantes extranjeros que acuden a estos espacios no pueden apreciar en su globalidad los lugares que visitan por la barrera idiomática con la que se encuentran; por eso, estimamos que la tarea de mediación que realizará el alumnado de la EOI con este colectivo es muy importante.

Para adaptarnos a sus necesidades programamos y ofrecemos cuatro tareas de carácter cultural que se desarrollarán utilizando el alemán como lengua vehicular.

\begin{tabular}{lll}
\hline $1^{\text {a }}$ Tarea & - & Se presentará el Proyecto ApS a todos los miembros. \\
& - & Se constituirán tándems lingüísticos a partir de cuestionarios \\
& que se pasan entre los miembros de ambas instituciones para \\
& conocer afinidad y compatibilidad de cara a crear los tándems \\
Escenario: & lingüísticos. \\
$\begin{array}{l}\text { Dependencias } \\
\begin{array}{l}\text { EOI (aulas y } \\
\text { patio). }\end{array}\end{array}$ & $\begin{array}{l}\text { Se mostrará el Blog del proyecto donde serán dados de alta los } \\
\text { estudiantes de la EOI para que puedan crear contenidos y vayan } \\
\text { actualizado el Blog. }\end{array}$ \\
\hline
\end{tabular}


Sesión $1^{\text {a: }}: 135$

minutos

- Finalizaremos con una Jornada de Confraternización Germano-española donde ofreceremos una muestra gastronómica. Cada participante aportará algún producto/ plato típico de su país de origen. Cada comida, bebida o utensilio que se aporte deberá venir etiquetado con el nombre del producto en la lengua materna del participante (alemán o español). Se presentarán los platos utilizando la L2 y se facilitará la receta de los platos típicos españoles en alemán a los miembros del Fórum para que aprendan a cocinar platos típicos españoles.

$2^{\mathrm{a}}$ Tarea Se organizarán las visitas para los miembros de la Asociación actuando el alumnado de la EOI como guías al Centro de Interpretación del Vino y la Sal de Chiclana de la Frontera (Cádiz).

Escenario:

Centro de

Interpretación

del Vino y

la Sal

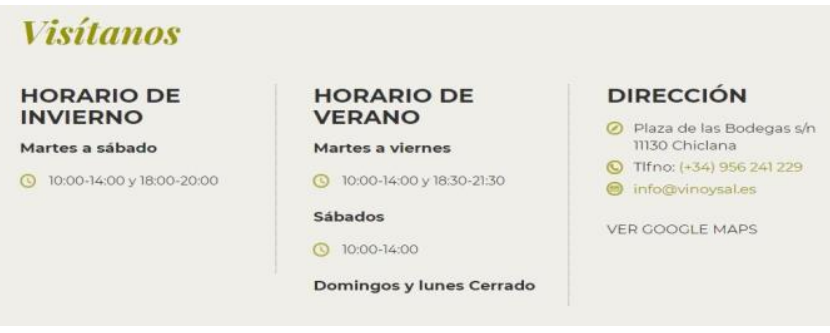

Imagen 3: Datos de contacto del Centro de Interpretación

Sesión 2a: 135 Como vemos la entrada a este centro es gratuita y tiene horario de tarde minutos compatible con el de la EOI. http://www.vinoysal.es

- Presentaciones en las salas del vino y sol

En este centro se relatan con paneles, aperos y fotografías el cultivo de la viña, así como la historia de las bodegas de Chiclana con distintos recursos audiovisuales. Además se muestra la crianza y envejecimiento del fino, el moscatel y otros vinos generosos de Chiclana, incluidos dentro de la Denominación de origen Jerez-XérèsSherry. 


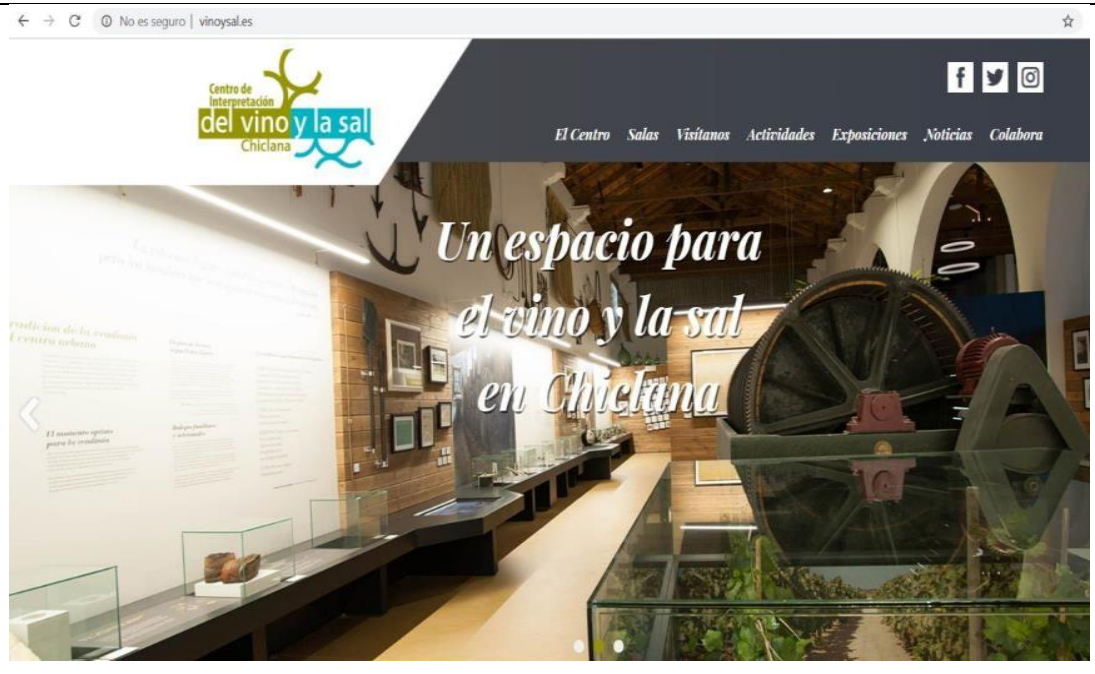

Imagen 4: Web del Centro de Interpretación para el vino y la Sal.

Se realizan 4 presentaciones por parte del alumnado de la EOI, de unos 10 minutos, relacionadas con las salas del vino sobre: 1. La viña, 2. La vendimia y 3 . Bodega y moscatel y la exposición correspondiente a la Sala del sol. Los participantes (miembros del Fórum y el resto del alumnado de la EOI) escucharán la exposición en la que se va ilustrando la visita cultural y posteriormente tendrán tiempo para ver la sala, el material de apoyo, hacer preguntas, etc.

Cada grupo habrá creado previamente un cuestionario sencillo de cinco preguntas que los asistentes cumplimentarán durante el transcurso de la exposición para asegurarse de que se está haciendo una escucha activa. Las preguntas son relevantes y tratan sobre los temas más significativos de la exposición.

$3^{\mathrm{a}}$ Tarea

Escenario:

Centro de Interpretación del Vino y la Sal
- Presentaciones en las salas del la sal y del mar.

Se realizan las 4 exposiciones de las Salas de la Sal sobre: 1.La marisma, 2. Salina artesanal y 3. La sal y el pescado de estero y otra presentación sobre la Sala del Mar. Se seguirá el mismo procedimiento seguido que en la actividad ApS segunda. 


\section{Sesión $3^{\mathrm{a}}: 135$} minutos
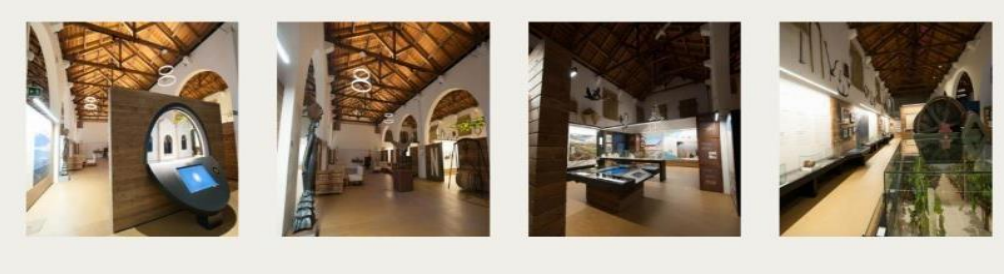

Imagen 5: Salas del centro de interpretación

$4^{\mathrm{a}}$ Tarea $\quad \bullet \quad$ Cata de vino y clausura

El centro ofrece la posibilidad de realizar talleres de cata y maridaje con

Escenario:

Centro de

Interpretación vinos de la tierra. Se realiza una cata de vino a modo de despedida y del Vino y la Sal

Sesión $4^{\text {a }}: 135$ clausura del proyecto donde el alumnado de la EOI actuarán como intérpretes.

minutos

Tras la cata tendremos una sesión de reflexión para valorar la experiencia e intercambiar opiniones, etc. El debate de reflexión y cierre es una de las partes más importantes del proyecto para comprobar el beneficio del servicio prestado, si los destinatarios ven útil y apropiada la experiencia y analizar los puntos fuertes y débiles de la experiencia.

Tabla 2: Resumen del servicio ofertado por el alumnado de la EOI. Elaboración propia.

- Tareas complementarias a las tareas ApS.

Para que el servicio prestado sea lo más satisfactorio posible y los beneficiarios de la actividad obtengan el mayor rendimiento, paralelamente a los servicios y de forma continuada durante los tres meses que dure la experiencia contarán con:

Tándem linguiístico Tándem lingüístico. (Miembro Fórum + Alumno/a EOI)

Intercambiarán correos electrónicos sobre curiosidades culturales sobre países germano hablantes y la provincia de Cádiz o cualquier tema de interés vinculado con la cultura española al menos una vez a la semana. Todos los correos electrónicos se redactarán en alemán por parte del alumnado de la EOI. Los 
miembros del Fórum podrán escribir sus correos en alemán o en español según prefieran.

Blog del ApS

El alumnado de la EOI facilitará la asimilación de contenidos culturales a través de aportaciones en el Blog:

- Glosario alemán-español: Cada grupo de trabajo creará un glosario de al menos 15 términos específicos sobre el tema que tendrá que presentar en el Centro de Interpretación del Vino y la Sal y publicarlo en el apartado correspondiente del blog para que pueda ser consultado por los miembros del Fórum. Si no existe una traducción exacta del término que se pretende introducir en el glosario, se debe escribir una breve descripción del término en alemán. Se utilizarán fotos e ilustraciones para facilitar la comprensión del léxico específico.

- Cada grupo creará un guion con los puntos que abordará en su presentación. El guion estará escrito en alemán exceptuando los términos autóctonos que se han explicado en el glosario y que aparecerán en cursiva.

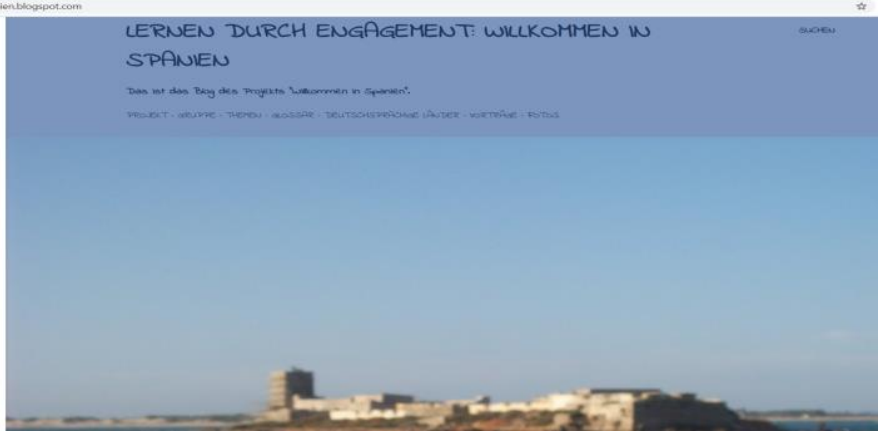

Imagen 6: Blog del ApS.

Material visual de apoyo
Cada grupo preparará, folletos, paneles y carteles de apoyo en alemán que puedan ayudar a enriquecer y facilitar las exposiciones.

Durante todo el proceso, deberán grabar y fotografiar el desarrollo de las actividades y publicar las fotografías y vídeos en el blog con notas explicativas.

Tabla 3: Tareas complementarias al servicio ofrecido. Elaboración propia. 


\section{Conclusiones}

Creemos que este proyecto ApS viene a cubrir la ausencia de tradición en proyectos de innovación educativa de estas características que nos encontramos en las Escuelas Oficiales de Idiomas y que generaría la posibilidad de establecer aprendizajes en contextos reales fuera del aula. Este es un modelo replicable que puede ser fácilmente adaptable a otras comunidades de extranjeros que viven en España facilitándoles así el establecimiento de vínculos con las distintas EOIs que se encuentran diseminadas por todo el territorio nacional y que ofrecen docencia en distintas lenguas extranjeras.

Este ApS permite el trabajo en red de una institución educativa y una entidad social facilitando que las EOIs puedan abrirse al entorno social.

Pretendemos proponer un servicio real efectivo a residentes extranjeros que deseen integrarse y acrecentar su conocimiento sobre nuestro país, nuestra cultura y costumbres pero que se ven limitados por la barrera idiomática. Damos así respuesta a una necesidad social que surge de la comunidad.

El servicio prestado a la asociación está equilibrado con la adquisición de conocimientos por parte del alumnado de la EOI proporcionándoles la oportunidad de comunicarse con personas que no forman habitualmente parte de su círculo y de su entorno.

Este tipo de metodología educativa ayudará al alumnado de la EOI a entrar en contacto con una comunidad social y lingüísticamente distinta que le facilitará el uso de la lengua extranjera con nativos en una situación comunicativa auténtica.

El servicio prestado mejorará significativamente el aprendizaje del alumnado dándole sentido, aportándole realismo, experiencia vital y permitiéndole adquirir nuevos aprendizajes. El alumnado se sentirá especialmente satisfecho por haber podido prestar un servicio a la comunidad, fomentando la convivencia y aumentado su responsabilidad cívica.

Uno de los aspectos más relevantes de este proyecto es que contribuirá a desarrollar competencias cívicas y sociales ayudando a desterrar actitudes xenófobas hacia migrantes y residentes extranjeros en nuestro país favoreciendo el respeto por otros pueblos y culturas.

\section{Referencias}

Alcaraz, S.; Chaparro, A. y García, B. (2016). Aprendizaje Servicio en la formación inicial de maestros y maestras de Educación Infantil. En La educación infantil en la sociedad del conocimiento. (pp. 341-349). Málaga: Universidad de Málaga.

Araujo Portugal, J.C. (2018). Las enseñanzas especializadas de idiomas de las Escuelas Oficiales de Idiomas: presente y futuro. e-Co. Revista Digital de Educación y Formación del Profesorado, (n⿳亠口冋 15), pp. 2-24. CEP de Córdoba y Consejería de Educación de la Junta de Andalucía. 
Battle, R. (s.f.). Guía práctica de aprendizaje-servicio. Proyecto social. Madrid. Santillana. Educación, S.L. Recuperado el 20 de diciembre, 2019, de https://cutt.ly/Oyo24fN

Cancelas y Ouviña, L.P. (2014). La formación inicial del profesorado de las Escuelas Oficiales de Idiomas. Lenguaje y Textos, (39), pp. 19-28.

Chupp, M.G. y Joseph, M.L. (2010). Getting the Most Out of Service Learning: Maximizing Student, University and Community Impact. Journal of Community Practice, (18:2), pp. 190-212.

Corredera, R. M. (2005). Atención a la diversidad en el aula de inglés en la EOI. Aldadis.Net: La Revista de Educación (7), pp. 41-49.

Díaz Ortega, H. (2005). Propuesta de estrategias de atención a la diversidad para la enseñanza de alemán en una Escuela Oficial de Idiomas. Aldadis.Net: La Revista de Educación (5), pp. 32-36.

Domingo Roget, A. y Balaguer Fábregas, C. (2017). Una experiencia de aprendizaje de Servicio en Colombia: conceptualización y estructuración. En Transformar para Educar 4. Aprendizaje Servicio (pp.16-40). Bogotá: Universidad del Norte.

Escobar, F. (1978). Principios del aprendizaje del adulto en programas de educación continua. Educación Médica y Salud 12 (2), pp.135-148.

García Ortiz, E. M. (2013). Experiencias multiculturales con la metodología del aprendizajeservicio. ELE: educación reglada y no reglada, pp. 61-75.

García Romero, D. y Lalueza Sazatornil, J.L. (2019). Procesos de Aprendizaje en Aprendizaje-Servicio Universitario: una revisión teórica. Educación XXI: Revista de la Facultad de Educación, (Vol.22), № 2, pp. 45-68.

Gezuraga Amundarain, M. y García-Pérez, A. (2018). ABProblemas, ABProyectos, ABretos... ¿Legado de Dewey? Valor añadido del ApS respecto a otros aprendizajes experienciales. En El Aprendizaje-Servicio en la Universidad. Una metodología docente y de investigación al servicio de la justicia social y el desarrollo sostenible. (pp. 22-27). Salamanca: Comunicación Social. Ediciones y Publicaciones.

González Enríquez, C. (2019). Inmigración en España: una nueva fase de llegadas, en Análisis del Real Instituto Elcano, (nº 28), pp. 1-9.

Martín, X. (2015). El aprendizaje servicio articula tres dinamismos educativos básicos: necesidades, servicios y aprendizaje. En 11 Ideas Clave ¿Cómo realizar un proyecto de aprendizaje servicio? (pp. 29-46). Barcelona: Graó. 
Martínez Lozano, V. y otros (Eds.) (2018). El Aprendizaje-Servicio en la Universidad. Una metodología docente y de investigación al servicio de la justicia social y el desarrollo sostenible. Salamanca: Comunicación Social. Ediciones y Publicaciones.

Mayor Paredes, D. (2013). Aprender realizando una actividad de utilidad social. Revista de Educación Social, (16), pp. 1-14.

Monreal-Gimeno, C. Marco Macarro, M. J., y Amador Muñoz, L. V. (2001). El adulto: etapas y consideraciones para el aprendizaje. Eúphoros, (3), pp. 97-112.

Monterrey, T. (2003). Los estudios ingleses en España (1900-1950): legislación curricular. Atlantis, (25)1, pp. 63-80.

Puig Rovira, J.M. (Coord.) (2015). 11 Ideas Clave ¿Cómo realizar un proyecto de aprendizaje servicio? Barcelona: Graó.

Puig Rovira, J. M., Battle, R., Bosch, C., y Palos, J. (2007). Aprendizaje servicio: Educar para la ciudadanía. Barcelona: Octaedro. 